\title{
Habilidade de leitura em alunos com TEA da rede pública de ensino do município de Curitiba/PR
}

\author{
Reading skills in students with ASD from public schools in Curitiba/PR \\ Capacidad de lectura en estudiantes con TEA de escuelas públicas en \\ Curitiba/PR
}

Karize Younes Barberini Perim

Mestra pela Universidade Presbiteriana Mackenzie, São Paulo, São Paulo, Brasil. kakayb@hotmail.com

ORCID - https://orcid.org/0000-0003-2333-4508

Marina Monzani da Rocha

Professora doutora na Universidade Presbiteriana Mackenzie, São Paulo, São Paulo, Brasil.

marinamonzani@gmail.com

ORCID - https://orcid.org/0000-0003-4995-7906

Recebido em 12 de dezembro 2019

Aprovado em 30 de agosto de 2020

Publicado em 30 de setembro de 2020

\section{RESUMO}

Considerando que a aprendizagem das habilidades de leitura tem funções significativas na vida dos indivíduos, tais habilidades foram investigadas dentre alunos com Transtorno do Espectro Autista (TEA), matriculados no Ensino Fundamental I, em escolas públicas do município de Curitiba. Participaram da pesquisa exploratória transversal 40 crianças (8 a 12 anos) e seus respectivos pais ou responsáveis. Ter o diagnóstico de TEA registrado na secretaria de educação e confirmado pela escala $A B C$, frequência escolar de pelo menos $70 \%$ dos dias letivos e estar em sala de recursos foram os critérios de inclusão. Os alunos realizaram a Prova de Avaliação dos Processos de Leitura (PROLEC) e os pais responderam a um questionário para caracterização da criança e da família. Os resultados confirmam que crianças com TEA apresentam dificuldades no desenvolvimento de habilidades de leitura. Pontuação abaixo da média das crianças que possuem habilidades normais de leitura foram encontradas para todos os anos escolares, especialmente nas provas que exigiam compreensão do texto. Isso sugere que os alunos com TEA desenvolvem mais facilmente a memorização das letras e palavras do que a compreensão do significado delas, bem como dos significados das orações compostas com essas palavras. Entretanto, alguns alunos atingiram resultados semelhantes aos da amostra normativa da PROLEC, especialmente os mais velhos. Não foi encontrada diferença em função do nível socioeconômico dos participantes. As dificuldades no desenvolvimento de habilidades de leitura apresentadas pelas crianças com TEA indicam a necessidade de organização pedagógica feita pelos professores, para favorecer as habilidades e competências individuais.

Palavras-chave: TEA; habilidade de leitura; desenvolvimento. 
http://dx.doi.org/10.5902/1984686X41426

\section{ABSTRACT}

Considering that learning reading skills has significant functions in the lives of individuals, these skills were investigated among students with Autistic Spectrum Disorder (ASD) enrolled in Elementary School I in public schools in Curitiba. Participated in the crosssectional exploratory research 40 children (8 to 12 years old) and their respective parents or caretakers. The inclusion criteria were: diagnosis of ASD registered at the school and confirmed by the ABC scale, school attendance of at least $70 \%$ of school days, and use of afterschool resource room. The students were assessed using the Reading Process Assessment Test (PROLEC) and the parents answered a questionnaire to characterize the child and the family. The results confirm that children with ASD have difficulties in developing reading skills. In all school years students had below-average scores in comparison with children with normal reading skills, especially on tests that required text comprehension. This suggests that students with ASD develop the memorization of letters and words more easily than the comprehension of their meanings, as well as the meanings of sentences composed with those words. However, some students achieved results similar to the test normative sample, especially the older ones. Socioeconomic status had no effect on the results. The difficulties in the development of reading skills presented by children with ASD indicate the need for teachers' pedagogical organization to favor individual skills and competences.

Keywords: ASD; reading skills; development.

\section{RESUMEN}

Teniendo em cuenta que el aprendizaje de las habilidades de lectura tiene funciones importantes en la vida de las personas, estas habilidades se investigaron entre los estudiantes con Trastorno del Espectro Autista (TEA) matriculados en la escuela primaria públicas de Curitiba. Cuarenta niños (de ocho a 12 años) y sus respectivos padres os tutores participaron em la investigación exploratória transversal. Tenere el diagnóstico de TEA registrado em el departamento de educación y confirmado por la escala $A B C$, la asistencia a escola de al menos el $70 \%$ de los días y la inclusión en sala de recursos fueron los criterios de inclusión. Los estudiantes tomaron la Prueba de evaluación del proceso de lectura (PROLEC) y los padres respondieron un cuestionario para caracterizar al niño y la familia. Los resultados confirman que los niños con TEA tienen dificultades para desarrollar habilidades de lectura. Se encontraron puntajes por debajo del promedio para niños con habilidades normales de lectura, especialmente en las pruebas que requieren comprensión de texto. Esto sugiere que los estudiantes con TEA desarrollan la memorización de letras y palabras más fácilmente que comprender su significado, así como lo significado de las oraciones compuestas con esas palabras. Sin embargo, algunos estudiantes lograron resultados similares a la muestra normativa de la prueba, especialmente los más antiguos. No se encontraron diferencias en función del estado socioeconómico de los participantes. Las dificultades en el desarrollo de las habilidades de lectura presentadas por los niños con TEA indican la necesidad de una organización pedagógica hecha por los maestros, para favorecer las habilidades y competencias individuales.

Palabras clave: TEA; capacidad de lectura; desarrollo. 
http://dx.doi.org/10.5902/1984686X41426

\section{Introdução}

O número de crianças com necessidades educacionais especiais (NEE) matriculadas em escolas regulares é crescente. As NEEs abrangem necessidades não apenas relacionadas com deficiências, mas também com as altas habilidades/superdotados, crianças em situação de rua, crianças de população remota ou nômade, crianças de minorias étnicas ou culturais e crianças de áreas ou grupos desfavorecidos ou marginais com dificuldades educacionais especiais (UNESCO, 1994). A Política Nacional de Educação Especial na Perspectiva da Educação Inclusiva (BRASIL, 2008), influenciada pela Declaração de Salamanca (UNESCO, 1994), determina que os alunos com deficiência, transtornos globais do desenvolvimento e altas habilidades/superdotação devem estar incluídos na rede regular de ensino, recebendo Atendimento Educacional Especializado (AEE) no contraturno. Neste cenário, a presença de alunos com Transtorno do Espectro do Autismo $^{1}$ (TEA), em escolas regulares, aumentou de maneira expressiva (NUNES; AZEVEDO; SCHMIDT, 2013).

É direito de todo cidadão ter acesso à educação, conforme assegurado na Constituição Federal, artigo 205 (BRASIL, 2004). De acordo com a Lei 13.146, de inclusão da pessoa com deficiência, artigo $4^{\circ}$, assegura-se que "toda pessoa com deficiência tem direito à igualdade de oportunidades [...] e não sofrerá discriminação" (BRASIL, 2015). A partir da Lei 12.764, de 27 de dezembro de 2012, o TEA foi reconhecido como uma deficiência e à pessoa com TEA foi garantido o acesso à educação e ao ensino profissionalizante (BRASIL, 2012).

Contudo, é notório que a igualdade de oportunidades está muito distante e que são oferecidos sistemas escolares diferentes e desiguais, sendo necessária uma construção de igualdade de acesso (DUBET, 2004). Isso aparece de maneira clara quando são analisados os casos de alunos diagnosticados com TEA, cujos desempenhos acadêmicos têm sido considerados críticos em diferentes estudos publicados em nosso país (GOMES; MENDES, 2010; NUNES; AZEVEDO; SCHMIDT, 2013). Silva (2016), em sua pesquisa sobre a presença de alunos com TEA em salas regulares, enfatiza a pequena quantidade de estudos envolvendo o TEA e a área da educação escolar, bem como a dificuldade que as escolas apresentam em organizar-se de maneira suficientemente apropriada para atender os alunos com alguma deficiência. Silva (2016) ressalta, ainda, que são poucas as escolas que já conseguiram tornar o ensino de qualidade acessível a este público. 
http://dx.doi.org/10.5902/1984686X41426

Compreendendo a necessidade de maiores conhecimentos sobre o TEA e sobre as possibilidades de ensino-aprendizagem no ambiente escolar, bem como sobre a maneira de tornar a aprendizagem significativa e satisfatória para esse público, o presente artigo propõe-se investigar o aprendizado de crianças com TEA inseridas em escolas municipais a partir da avaliação da habilidade de leitura.

A aprendizagem das habilidades de leitura favorece a compreensão dos estímulos que estão a sua volta, a relação com outras pessoas, bem como a aproximação aos conteúdos escolares, ou seja, ela tem funções significativas na vida dos indivíduos (GOMES; CARVALHO; SOUZA, 2014). Problemas na leitura podem impedir que os indivíduos desenvolvam outras habilidades, como, por exemplo, o domínio da linguagem, o crescimento do vocabulário, a escrita e o conhecimento das palavras, o que irá repercutir no desenvolvimento de aprendizagens posteriores (CUETOS; RODRIGUES; RUANO, 2015).

Problemas de aquisição de leitura são registrados em diversos países do mundo (NUNES; WALTER, 2016). Ainda que, no Brasil, os estudos sejam incipientes, entre 30\% e $40 \%$ dos alunos nos primeiros anos do Ensino Fundamental (EF) evidenciam dificuldades na aprendizagem da leitura (ANDRADE et al., 2014). Pouco sabe-se sobre a dificuldade de compreensão e leitura da população com TEA e poucos programas interventivos de leitura, voltados especificamente a eles, estão disponíveis (NUNES; WATER, 2016). Isso dá-se, possivelmente, em função das estratégias pedagógicas utilizadas pelos professores terem produzido poucos efeitos de aprendizagem acadêmica desses alunos, incluindo a habilidade de leitura e escrita (NUNES; AZEVEDO; SCHMIDT, 2013).

Gomes e Mendes (2010) investigaram alunos com o diagnóstico de TEA, matriculados em escolas regulares no município de Belo Horizonte, no ano de 2008, por meio de entrevistas com os professores desses alunos. O estudo contou com a participação de 33 professores e 23 alunos com TEA, sendo 5 da Educação Infantil (EI) e 18 do EF, representando aproximadamente $50 \%$ do total de alunos com esse diagnóstico registrado, matriculados no ensino regular daquela cidade no ano da pesquisa. No que diz respeito à aprendizagem de habilidades pedagógicas básicas, observou-se que apenas 10\% do total de alunos investigados no EF sabiam ler, escrever, fazer contas e acompanhar os conteúdos pedagógicos desenvolvidos pelas escolas. Para Gomes (2011), tais resultados podem ser decorrentes do fato dessa população apresentar dificuldades em aprender por 
http://dx.doi.org/10.5902/1984686X41426

métodos convencionais de ensino e precisar com frequência de metodologias mais adequadas.

Pessoas com TEA podem ser beneficiadas pela aprendizagem de habilidades de leitura, permitindo-Ihes maior compreensão e interação com o meio, mas esse processo de ensino de habilidades de leitura pode ser uma tarefa difícil e desafiadora, pois, além das habilidades serem complexas, pessoas com TEA podem apresentar dificuldades em aprender pelo método convencional de ensino, precisando, assim, uma adequação pedagógica (GOMES; CARVALHO; SOUZA, 2014).

Considerando que a aprendizagem da leitura é um processo complexo (HANNA et al., 2010), há duas perspectivas diferentes na literatura a respeito dessa aprendizagem por pessoas com TEA (NATION et al., 2006). A primeira considera que o repertório pobre de habilidades de linguagem, o qual é típico do quadro de TEA, coloca essas pessoas em grande risco de fracasso escolar na aprendizagem desses conteúdos. Já a segunda descreve sucessos no ensino da leitura em indivíduos com TEA, ainda que os pesquisadores realcem a necessidade do cuidado com a generalização dos resultados de estudos de caso com essa população, principalmente pela extensa variabilidade no repertório de habilidades cognitivas e de linguagem observadas (NATION et al., 2006).

Gomes e Souza (2016), pesquisando o ensino de sílabas simples, leitura combinatória e leitura com compreensão para crianças com TEA, concluíram que o procedimento de ensino utilizado foi efetivo para promover a aprendizagem de leitura combinatória com compreensão para esses alunos. Participaram do estudo 3 crianças com diagnóstico de TEA leve/moderado, estudantes de escolas comuns, que inicialmente foram avaliados pela Childhood Autism Rating Scale - CARS (SCHOPLER; REICHLER; RENNER, 1988), pelo Psychoeducational Profile-Revised - PEP-R (SCHOPLER et al., 1990) e pelo Assessment of Basic Learning Skills - ABLA (KERR et al., 1977).

Gomes (2011) relata que, quando as pessoas com TEA aprendem a ler, existe uma diferença relevante no desempenho, no que diz respeito à leitura oral do texto, sem compreender necessariamente o que está escrito nele, e à leitura com compreensão, o que exige indispensavelmente o entendimento do conteúdo que está expresso no texto. A autora complementa afirmando que desempenhos na leitura oral são melhores do que na compreensão do texto (GOMES, 2011).

Um exemplo dessa diferença é ilustrado no estudo de Nation et al. (2006), o qual teve como objetivo principal investigar as habilidades de leitura de crianças e adolescentes 
http://dx.doi.org/10.5902/1984686X41426

diagnosticados com TEA, analisando quatro componentes: a leitura oral de palavras impressas, leitura oral de pseudopalavras, leitura de texto com compreensão e, por fim, a precisão da leitura oral de textos. Esse estudo contou com a participação de 41 indivíduos com TEA, os quais eram falantes e tinham idades entre 6 e 15 anos. Os resultados indicaram desempenhos médios na avaliação de leitura oral de palavras, pseudopalavras e leitura oral de textos, no entanto, houve desempenho baixo dos participantes com relação a leitura de textos com compreensão. De maneira geral, o estudo indicou problemas na leitura de indivíduos com TEA (NATION et al., 2006).

Silva (2011), em sua pesquisa sobre a aprendizagem da leitura e da escrita em crianças com TEA, com o intuito de contribuir nas práticas de ensino e promover estratégias pedagógicas para alcançar o sucesso educativo dessas crianças, observou que a melhor maneira de ensinar é utilizando vários métodos ou um método misto de aprendizagem e concluiu que há alunos com TEA que escrevem, mas que não conseguem ler o que escreveram. Ainda que seja um estudo realizado com apenas 3 crianças, o trabalho de Silva (2011) apresenta questões importantes sobre o aprendizado da leitura e escrita em crianças com TEA.

Os programas de leitura, que visam estratégias baseadas em evidência recomendadas pelo Conselho Nacional de Pesquisa em Leitura (NATIONAL RESEARCH READING PANEL [NRP], 2000), têm apresentado efeitos promissores no desempenho de alunos com desenvolvimento típico nos últimos anos (EL ZEIN et al., 2014). Estudos dirigidos por Otaiba e Fuchs (2002) sinalizam, no entanto, que essas metodologias são pouco efetivas para as pessoas diagnosticadas com TEA. Em seus estudos, apenas 50\% desses alunos apresentam avanços quando expostos a essas estratégias (AL OTAIBA; FUCHS, 2002).

Diante disso, fica clara a importância de encontrar métodos adequados a cada criança, conforme os estudos de Gomes, Carvalho e Souza (2014), que apontam em sua pesquisa, os aspectos relevantes do ensino de leitura para pessoas com TEA, no sentido de que essa população pode apresentar dificuldades em aprender esse conteúdo quando são ensinados utilizando um método de ensino convencional.

Tendo como base os estudos acima citados, o presente artigo tem como objetivo verificar se alunos com TEA do município de Curitiba com idades entre 8 e 12 anos matriculados em escola municipal regular apresentam habilidade de leitura, bem como 0 grau de habilidade de leitura daqueles que leem e, por fim, verificar se há relação entre as 
http://dx.doi.org/10.5902/1984686X41426

variáveis idade e nível socioeconômico e os diferentes níveis de leitura, de maneira a pensar sobre a necessidade de alternativas de ensino diferentes para cada subgrupo.

\section{Método}

Foi realizada uma pesquisa do tipo exploratória transversal, que teve como universo o ensino fundamental de 13 escolas do EF I da secretaria municipal da cidade de Curitiba/PR. Esse desenho foi escolhido em função da pesquisa exploratória proporcionar mais informações sobre o assunto a ser investigado, possibilitando definição e delineamento, permitindo também o estudo do tema sob diversos ângulos e aspectos (PRODANOV; FREITAS, 2013).

O projeto foi aprovado pelo Comitê de Ética da Prevent Senior sob o protocolo (1.984.340). Todas as orientações preconizadas na resolução 466 de 12 de dezembro de 2012 do Conselho Nacional de Saúde foram garantidas durante a execução do estudo.

\section{Participantes}

Participaram 40 crianças diagnosticadas com TEA, dentre elas 33 do sexo masculino e 7 do sexo feminino, bem como seus respectivos pais ou responsáveis, totalizando 80 participantes. Foram considerados como critérios de inclusão para as crianças participarem nesse estudo: ter registrado na secretaria de educação do município o diagnóstico de TEA, pertencer à faixa etária de f8 a 12 anos), apresentar a frequência na escola de pelo menos $70 \%$ dos dias letivos e estarem inseridos nas salas de recursos. Foram excluídos da amostragem os alunos com TEA que frequentavam a escola em horário reduzido.

\section{Instrumentos}

A Prova de Avaliação dos Processos de Leitura (PROLEC) (CUETOS; RODRIGUES; RUANO, 2015) foi o instrumento utilizado para a avaliação da habilidade de leitura das crianças. Esta é composta por quatro blocos distribuídos para a avaliação de 4 processos de leitura: identificação de letras, processos léxicos, sintáticos e semânticos (OLIVEIRA et al., 2013), que tem como finalidade avaliar e definir os processos de leitura, procurando estabelecer um consenso entre os procedimentos utilizados para definir o perfil dos estudantes quanto ao seu desempenho nos processos de leitura e seus preditores para a alfabetização (OLIVEIRA et al., 2011). Por meio dela, é possível compreender as dificuldades que existem na leitura, bem como obter auxílio na análise do diagnóstico dos 
http://dx.doi.org/10.5902/1984686X41426

transtornos de aprendizagem (CUETOS; RODRIGUES; RUANO, 2015). É importante ressaltar que, para que todos os alunos participantes da pesquisa pudessem responder a esta prova, foi necessário adaptar o material, ajustando a escrita para letras em caixa alta.

Para os pais ou responsáveis, foi utilizado um questionário sociodemográfico, elaborado para a realização da pesquisa com perguntas relacionadas ao diagnóstico da criança, tempo que levou para chegar a esse diagnóstico, serviços e profissionais que a criança tem acesso e uso de medicamentos. Em conjunto com essas questões, foi apresentado o "Critério Padrão de Avaliação Socioeconômica Brasil" estabelecido pela ABEP (Associação Brasileira de Empresas de Pesquisa), que procura avaliar o poder de consumo da família a partir dos itens de conforto presentes na residência, tipo de água utilizada, pavimentação do trecho da rua onde mora e grau de instrução do chefe da família. Foram estabelecidos 6 estratos socioeconômicos: A, B1, B2, C1, C2, D-E. Para facilitar a análise dos resultados, em função do nível socioeconômico no presente estudo, os estratos A e B1 foram denominados como nível Alto, os estratos B2 como nível Médio e os estratos C1, C2 e D-E como nível Baixo.

Por fim, os pais ou responsáveis dos alunos também responderam ao Inventário de Comportamento da Criança Autista (Autism Behavior Checklist - ABC; KRUG; ARICK; ALMOND, 1980), instrumento adaptado por Marteleto e Pedromônico (2005) para uso no Brasil. É voltado para o rastreio do TEA a partir de 57 itens de comportamentos atípicos e não adaptativos, que são pontuados de 1 a 4 (TAMANAHA; MARTELETO; PARISSINOTO, 2014). Esse instrumento foi utilizado para a confirmação do diagnóstico do TEA.

\section{Procedimentos}

A partir da autorização da Secretaria Municipal de Educação do município, foi estabelecido o contato com as escolas que atendiam os alunos com TEA em sala de recurso multifuncional para realização da pesquisa. Com autorização da direção das escolas, os pais ou responsáveis pelos alunos com TEA foram convidados a permanecer na escola para um diálogo com a pesquisadora, explicando o objetivo da pesquisa e apresentando o teste que seria aplicado nas crianças. A primeira etapa da coleta de dados envolveu os pais ou responsáveis preencherem o TCLE autorizando a participação, o questionário sobre o histórico do diagnóstico das crianças e os dados sociodemográficos da família e o $A B C$, para verificação do diagnóstico do TEA, o qual os pais ou responsáveis responderam sozinhos, após a explicação da pesquisadora. Na segunda etapa, foi 
http://dx.doi.org/10.5902/1984686X41426

realizada a avaliação das crianças com a PROLEC. Inicialmente, solicitou-se o assentimento verbal, com a apresentação do teste para certificar de que os alunos aceitariam participar e, na sequência, foi aplicada a prova na própria sala de recursos multifuncionais, na qual o aluno tem o atendimento. Houve aceitação de $100 \%$ dos alunos para realizar o teste e a aplicação levou em média 12 minutos para cada criança.

Os testes PROLEC e ABC foram corrigidos de acordo com os seus respectivos manuais. Inicialmente, foi verificado quantos alunos conseguiram responder às provas da PROLEC e o nível de habilidade de leitura apresentado em cada uma delas. Em seguida, foram comparadas as médias obtidas pelos participantes em cada uma das provas com as médias da amostra normativa da PROLEC. Essa análise foi realizada separadamente por ano escolar usando o teste t de amostra única. Por fim, o teste qui-quadrado foi utilizado para verificar se havia diferença na proporção das classificações "normal", "dificuldade" e "dificuldade grande" nas provas da PROLEC em função das variáveis: faixa etária (8 a $9 \mathrm{x}$ 10 a 12 anos) e nível socioeconômico (baixo, médio e alto). As análises estatísticas foram realizadas utilizando o software SPSS 21.0. Foram considerados significativos valores de $p$ menores ou iguais a 0,05 .

\section{Resultados e discussão}

A PROLEC apresenta 3 categorias normativas para a interpretação dos resultados, bem como médias e escalas de pontuação para cada prova, considerando o nível escolar. A partir dessa avaliação é possível identificar se há dificuldade em alguns dos processos e se esta é grande ou pequena (CUETOS; RODRIGUES; RUANO, 2015). As 3 categorias apresentadas pela PROLEC são: $\mathrm{N}$ - normal; D - dificuldade; DD - dificuldade grande. $\mathrm{Na}$ Tabela 1 , serão apresentados os resultados gerais referentes às provas realizadas. 
http://dx.doi.org/10.5902/1984686X41426

Tabela 1 - Resultados e categorias gerais dos alunos participantes com relação à PROLEC

\begin{tabular}{lcc}
\hline Provas & $\begin{array}{c}\text { Quantidade de alunos que } \\
\text { responderam a prova }\end{array}$ & N (\%) \\
\hline Nome ou som das letras & $38(95,0 \%)$ & $21(52,5 \%)$ \\
$\quad$ Normal & & $10(25,0 \%)$ \\
$\quad$ Dificuldade & $21(52,5 \%)$ & $9(22,5 \%)$ \\
$\quad$ Dificuldade grande & & \\
Igual-diferente & & $10(25,0 \%)$ \\
$\quad$ Normal & & $5(12,5 \%)$ \\
$\quad$ Dificuldade & $18(45,0 \%)$ & $25(62,5 \%)$ \\
$\quad$ Dificuldade grande & & \\
Leitura de pseudopalavras & & $10(25,0 \%)$ \\
$\quad$ Normal & & $1(2,5 \%)$ \\
$\quad$ Dificuldade & $7(17,5 \%)$ & $29(72,5 \%)$ \\
Dificuldade grande & & $4(10,0 \%)$ \\
Estruturas gramaticais & & $2(5,0 \%)$ \\
$\quad$ Normal & & $34(85,0 \%)$ \\
Dificuldade & & \\
Dificuldade grande & & $3(7,5 \%)$ \\
Compreensão de textos & $(17,5 \%)$ & $2(5,0 \%)$ \\
$\quad$ Normal & & $35(87,5 \%)$ \\
$\quad$ Dificuldade & & \\
$\quad$ Dificuldade grande & &
\end{tabular}

Fonte: As autoras (2020).

A prova na qual a maior parte dos participantes (52,5\%) obteve pontuação normal foi a prova 1 "Nome ou som das letras". As provas "Estruturas Gramaticais" e "Compreensão de Textos" foram as quais mais alunos obtiveram pontuação equivalente a Dificuldade Grande: $85 \%$ e $87,5 \%$, respectivamente, mostrando que os participantes da presente amostra sabem identificar as letras, mas apresentam grande dificuldade para a compreensão do significado da oração. Deve-se atentar ao fato de que apenas $17,5 \%$ dos participantes conseguiu responder à última prova da PROLEC, que avalia essa habilidade, e somente 3 tiveram um resultado dentro do esperado em comparação com a amostra normativa da prova, o que representa 7,5\% dos alunos avaliados, indicando grande dificuldade dentre os participantes na extração do significado, integração na memória e elaboração de inferências. Esse resultado converge com o achado de Nation et al. (2006), o qual encontrou também um desempenho baixo dos alunos do TEA no que se refere à leitura de textos com compreensão. Os resultados podem ser decorrentes do fato de que as crianças com TEA apresentam dificuldades para comunicar-se e para interagir socialmente e essas dificuldades afetam o uso da língua falada. Com isso, a aprendizagem 
da leitura para essas crianças fica comprometida, pois aprender a ler, quando não se tem domínio do idioma falado, é uma tarefa bem mais complexa (SAMPAIO; OLIVEIRA, 2017).

Ainda que se tenha dificuldades para entender as orações, elas também são pouco informativas e não aparecem isoladas, sendo que formam parte de um contexto em que discorre a ação e dá sentido à oração. Se a integração da informação na memória é considerada uma tarefa importante no processo de leitura, não deve ser a última, visto que essa informação armazenada deve servir para enriquecer o conhecimento e não para incrementar passivamente a quantidade de informações armazenadas (CUETOS; RODRIGUES; RUANO, 2015). Gomes e Mendes (2010), trabalhando com alunos com TEA de Belo Horizonte, encontraram que apenas $10 \%$ destes sabiam ler. No presente estudo, $17,5 \%$ dos participantes tiveram habilidades para responder a prova de compreensão de texto e apenas 7,5\% obtiveram um resultado normal em comparação com a amostra normativa do teste utilizado. Ambos os resultados evidenciam a dificuldade para 0 desenvolvimento de habilidades de leitura por crianças com TEA. Santos, Zacarias e Barbosa (2015) relatam, em sua pesquisa sobre a aprendizagem e o TEA, que há peculiaridades na aprendizagem de cada indivíduo em decorrência do modo de compreensão e do nível de TEA que cada criança apresenta. Diante disso, métodos utilizados na alfabetização de alguns alunos podem não servir para outros, sendo necessário o desenvolvimento de estratégias diferenciadas para atender a todos e possibilitar o processo de ensino-aprendizagem. Em concordância com Gomes (2015), as autoras enfatizam que cada conteúdo trabalhado deve ser transmitido às crianças com TEA em pequenas porções para que haja uma compreensão satisfatória, pois, o processo de assimilação dessas crianças é, geralmente, lento (SANTOS; ZACARIAS; BARBOSA, 2015).

A Tabela 2 apresenta os resultados obtidos pelos participantes nas provas da PROLEC divididos por ano escolar. As médias obtidas foram comparadas com a amostra normativa do instrumento para análise do desempenho dos participantes em cada uma das habilidades estudadas usando o teste t para amostras únicas. 
Tabela 2 - Resultados obtidos pelos participantes do $2^{\circ}$ ao $5^{\circ}$ ano na PROLEC

\begin{tabular}{|c|c|c|c|c|c|c|c|}
\hline & PROLEC & Min. & Máx. & Média (DP) & $\begin{array}{c}\text { PROLEC } \\
\text { Média (DP) }\end{array}$ & Diferença & $p$ \\
\hline & Nome ou som das letras & 7 & 11 & $8,67(2,08)$ & $19,37(0,88)$ & $-10,70$ & 0,012 \\
\hline $2^{\circ}$ & Igual - diferente & 0 & 20 & $11,33(10,26)$ & $19,89(1,26)$ & $-8,56$ & 0,286 \\
\hline ANO & Leitura de pseudopalavras & 0 & 0 & $0(0)$ & $27,44(3,20)$ & $-27,44$ & - \\
\hline \multirow[t]{2}{*}{$N=3$} & Estruturas gramaticais & 0 & 0 & $0(0)$ & $9,05(2,55)$ & $-9,05$ & - \\
\hline & Compreensão de textos & 0 & 0 & $0(0)$ & $9,83(3,87)$ & $-9,93$ & - \\
\hline \multirow{5}{*}{$\begin{array}{c}\text { 3o } \\
\text { ANO } \\
\mathrm{N}= \\
16\end{array}$} & Nome ou som das letras & 7 & 20 & $15,81(6,53)$ & $19,31(0,88)$ & $-3,50$ & 0,049 \\
\hline & Igual - diferente & 0 & 20 & $5,13(7,18)$ & $18,89(2,64)$ & $-13,76$ & 0,000 \\
\hline & Leitura de pseudopalavras & 0 & 30 & $4,63(9,81)$ & $26,64(3,14)$ & $-22,31$ & 0,000 \\
\hline & Estruturas gramaticais & 0 & 7 & $0,44(1,75)$ & $11,40(2,33)$ & $-10,96$ & 0,000 \\
\hline & Compreensão de textos & 0 & 8 & $0,50(2,00)$ & $11,46(3,76)$ & $-10,96$ & 0,000 \\
\hline \multirow{5}{*}{$\begin{array}{c}\mathbf{4}^{\circ} \\
\text { ANO } \\
\mathrm{N}= \\
16\end{array}$} & Nome ou som das letras & 17 & 20 & $18,81(1,22)$ & $19,90(0,30)$ & $-1,09$ & 0,003 \\
\hline & Igual - diferente & 0 & 20 & $14,19(7,70)$ & $19,64(0,60)$ & $-5,45$ & 0,013 \\
\hline & Leitura de pseudopalavras & 0 & 30 & $12,94(13,73)$ & $28,39(2,35)$ & $-15,45$ & 0,000 \\
\hline & Estruturas gramaticais & 0 & 15 & $1,44(4,13)$ & $11,97(1,47)$ & $-10,53$ & 0,000 \\
\hline & Compreensão de textos & 0 & 13 & $1,3(3,40)$ & $13,78(1,52)$ & $-12,66$ & 0,000 \\
\hline \multirow{5}{*}{$\begin{array}{c}\mathbf{5}^{\circ} \\
\text { ANO } \\
\mathrm{N}=5\end{array}$} & Nome ou som das letras & 18 & 20 & $19,40(0,89)$ & $19,97(0,17)$ & $-0,57$ & 0,227 \\
\hline & Igual - diferente & 10 & 19 & $15,40(4,16)$ & $19,37(1,33)$ & $-3,97$ & 0,100 \\
\hline & Leitura de pseudopalavras & 24 & 30 & $28,20(2,68)$ & $27,99(2,34)$ & 0,21 & 0,870 \\
\hline & Estruturas gramaticais & 0 & 13 & $9,00(5,20)$ & $12,04(1,89)$ & $-3,04$ & 0,261 \\
\hline & Compreensão de textos & 0 & 14 & $9,60(5,55)$ & $14,01(1,76)$ & $-4,41$ & 0,150 \\
\hline
\end{tabular}

Legenda: Min. = Pontuação mínima Máx. = Pontuação Máxima

Fonte: As autoras (2020).

Os participantes que estavam no $2^{\circ}$ ano obtiveram resultados abaixo da média dos escolares que se encontram dentro da categoria normal de leitura para as provas "Nome ou som das letras" e "Igual - diferente". Além disso, nenhum participante do $2^{\circ}$ ano teve habilidades para responder às outras provas da PROLEC. Esse é um indício de que as crianças com TEA, no início do ciclo de aprendizagem, apresentam dificuldades no desenvolvimento da leitura acima do esperado na comparação com seus pares que apresentam desenvolvimento típico (amostra normativa PROLEC - CUETOS; RODRIGUES; RUANO, 2015).

Tanto os participantes do $3^{\circ}$ quanto os do $4^{\circ}$ ano obtiveram, em média, resultados abaixo da média das crianças que possuem habilidades normais de leitura. Já os participantes que frequentavam $05^{\circ}$ ano não obtiveram, em média, resultados estatisticamente diferentes de seus pares da amostra normativa da PROLEC.

Pode-se observar que, no geral, os alunos com TEA obtiveram resultados abaixo da média das crianças que possuem habilidades normais de leitura. Apenas os resultados obtidos pelas crianças que estavam no $5^{\circ}$ ano EF I foi mais próximo aos dos alunos deste ano escolar da amostra normativa da PROLEC. Esse resultado pode sugerir que, à medida que os anos escolares aumentam, os resultados são melhores, ou seja, um processo de 
http://dx.doi.org/10.5902/1984686X41426

desenvolvimento da habilidade de leitura pode ocorrer nas crianças com TEA que avançam efetivamente nos anos escolares. A pesquisa de Salles et al. (2013), sobre a leitura de palavras isoladas para crianças de $1^{\circ}$ ao $7^{\circ}$ ano, faz uma comparação das crianças mais escolarizadas com as menos escolarizadas e observa que aquelas em idade escolar maior também tiveram desempenho superior da leitura de palavras isoladas (LPI) ao das crianças com idade escolar menor em todas as categorias estudadas nessa pesquisa: total, regulares, irregulares e pseudopalavras. Da mesma forma, as crianças mais velhas apresentam desempenho superior em todas as categorias de LPI (SALLES et al, 2013). Esses resultados sugerem que, apesar de inicialmente as crianças com TEA apresentarem mais dificuldade do que seus pares na aquisição de habilidades de leitura, com o passar dos anos algumas delas podem alcançar seus pares no desenvolvimento dessas habilidades. No entanto, mesmo dentre as crianças que estavam no $5^{\circ}$ ano, encontramos algumas que não conseguiram responder adequadamente às provas que avaliam estruturas gramaticais e compreensão de texto. Além disso, é necessário fazer uma ressalva no que se refere à evasão escolar das crianças que apresentam dificuldades nos anos iniciais, de maneira que, para ser possível afirmar que o desenvolvimento de leitura acontece, mesmo que de maneira tardia dentre as crianças com TEA, é necessário fazer um estudo longitudinal. Nesse sentido, um estudo recente buscou compreender a trajetória dos alunos com TEA matriculados em um grande município do estado de São Paulo e evidenciou que apenas 12,92\% dos alunos fazem a trajetória completa nos anos escolares, o que sugere altos índices de evasão escolar (TALARICO; LAPLANE, 2016). Em outras palavras, é necessário enfatizar que poucas são as crianças com TEA que permanecem na escola, o que permite que se formule a hipótese de que aquelas que permanecem são justamente as com um quadro mais leve, com menor prejuízo nas habilidades cognitivas.

De qualquer forma, o resultado demonstra que a aprendizagem da leitura, ainda que lenta, parece ser possível para uma parte das crianças com TEA. De fato, a leitura é uma habilidade complexa, envolvendo inúmeros aspectos e etapas que devem ser ensinados, um a um, em pequenas fases, gradativamente, iniciando pelas habilidades mais simples às mais complexas (GOMES, 2015). Skinner (1972) afirma que, desde que sejam utilizados procedimentos adequados, pessoas com diagnóstico de TEA conseguem aprender, pois o ensino é uma combinação de contingências sob as quais as pessoas, independentemente de ter desenvolvimento atípico, aprendem. 
http://dx.doi.org/10.5902/1984686X41426

$\mathrm{Na}$ Tabela 3, são apresentados os resultados obtidos nos testes em função da faixa etária do participante. Eles foram divididos em dois grupos para que a comparação pudesse ser realizada: crianças mais novas - dos 8 aos 9 anos $(\mathrm{N}=21)$ - e crianças mais velhas dos 10 aos 12 anos $(\mathrm{N}=19)$.

Tabela 3 - Classificação do resultado das provas da PROLEC em função da faixa etária

\begin{tabular}{|c|c|c|c|c|c|}
\hline Prova PROLEC & Faixa Etária & Normal & Dificuldade & $\begin{array}{c}\text { Dificuldade } \\
\text { Grande }\end{array}$ & $p$ \\
\hline Nome ou Som das Letras & $\begin{array}{c}8 \text { a } 9 \text { anos } \\
10 \text { a } 12 \text { anos }\end{array}$ & $\begin{array}{c}9(42,9 \%) \\
12(63,2 \%)\end{array}$ & $\begin{array}{l}4(19,0 \%) \\
6(31,6 \%)\end{array}$ & $\begin{array}{c}8(38,1 \%) \\
1(5,3 \%)\end{array}$ & 0,045 \\
\hline Igual - diferente & $\begin{array}{c}8 \text { a } 9 \text { anos } \\
10 \text { a } 12 \text { anos }\end{array}$ & $\begin{array}{l}3(14,3 \%) \\
7(36,8 \%)\end{array}$ & $\begin{array}{c}1(4,8 \%) \\
4(21,1 \%)\end{array}$ & $\begin{array}{c}17(81,0 \%) \\
8(42,1 \%)\end{array}$ & 0,038 \\
\hline Leitura de pseudopalavras & $\begin{array}{c}8 \text { a } 9 \text { anos } \\
10 \text { a } 12 \text { anos }\end{array}$ & $\begin{array}{c}4(19 \%) \\
6(31,6 \%)\end{array}$ & $\begin{array}{c}0(0 \%) \\
1(5,3 \%) \\
\end{array}$ & $\begin{array}{c}17(81 \%) \\
12(63,2 \%)\end{array}$ & 0,338 \\
\hline Estruturas gramaticais & $\begin{array}{c}8 \text { a } 9 \text { anos } \\
10 \text { a } 12 \text { anos } \\
\end{array}$ & $\begin{array}{c}1(4,8 \%) \\
3(15,8 \%) \\
\end{array}$ & $\begin{array}{c}0(0 \%) \\
2(10,5 \%)\end{array}$ & $\begin{array}{l}20(95,2 \%) \\
14(73,7 \%) \\
\end{array}$ & 0,137 \\
\hline Compreensão de textos & $\begin{array}{c}8 \text { a } 9 \text { anos } \\
10 \text { a } 12 \text { anos }\end{array}$ & $\begin{array}{c}1(4,8 \%) \\
2(10,5 \%)\end{array}$ & $\begin{array}{c}0(0 \%) \\
2(10,5 \%)\end{array}$ & $\begin{array}{l}20(95,2 \%) \\
15(78,9 \%)\end{array}$ & 0,327 \\
\hline
\end{tabular}

Fonte: As autoras (2020).

O teste qui-quadrado indicou que houve diferença significativa na comparação dos grupos em duas provas da PROLEC: Nome ou som da letra $(p=0,045)$ e Igual - Diferente $(p=0,038)$. Em ambas, uma maior proporção de crianças mais novas (faixa etária dos 8 aos 9 anos) obtiveram resultados que foram classificados como dificuldade grande. Esse resultado é coerente com a progressão no resultado obtido nas provas da PROLEC na análise por ano escolar apresentada no presente estudo, bem como confirma o resultado de Salles et al. (2013), que indicou que as crianças mais velhas apresentaram melhores resultados em todas as provas realizadas.

Por fim, foram feitas análises para verificar diferença nos resultados da PROLEC em função do nível socioeconômico da família da criança com TEA. O nível socioeconômico foi dividido em três faixas: Alto $(\mathrm{N}=14)$, Médio $(\mathrm{N}=14)$ e Baixo $(\mathrm{N}=12)$. O teste qui-quadrado não indicou diferenças estatisticamente significativas nos resultados obtidos pelas crianças dos três grupos. 
http://dx.doi.org/10.5902/1984686X41426

Tabela 4 - Classificação do resultado das provas da PROLEC em função do nível socioeconômico

\begin{tabular}{lccccc}
\hline Prova PROLEC & $\begin{array}{c}\text { Nível } \\
\text { Socioeconômico }\end{array}$ & Normal & Dificuldade & $\begin{array}{c}\text { Dificuldade } \\
\text { Grande }\end{array}$ & $\boldsymbol{p}$ \\
\hline \multirow{3}{*}{ Nome ou Som das Letras } & Baixo & $6(50 \%)$ & $4(33,3 \%)$ & $2(16,7 \%)$ & \\
& Médio & $9(64,3 \%)$ & $3(21,4 \%)$ & $2(14,3 \%)$ & 0,592 \\
& Alto & $6(42,9 \%)$ & $3(21,4 \%)$ & $5(35,7 \%)$ & \\
\hline \multirow{3}{*}{ Igual - diferente } & Baixo & $4(33,3 \%)$ & $2(16,7 \%)$ & $6(50 \%)$ & \\
& Médio & $3(21,4 \%)$ & $2(14,3 \%)$ & $9(64,3 \%)$ & 0,829 \\
& Alto & $3(21,4 \%)$ & $1(7,1 \%)$ & $10(71,4 \%)$ & \\
\hline \multirow{2}{*}{ Leitura de pseudopalavras } & Baixo & $3(25 \%)$ & $0(0 \%)$ & $9(75 \%)$ & \multirow{2}{*}{0,733} \\
& Médio & $3(21,4 \%)$ & $1(7,1 \%)$ & $10(71,4 \%)$ & $0,70(71,4 \%)$ \\
\hline \multirow{2}{*}{ Estruturas gramaticais } & Alto & $4(28,6 \%)$ & $0(0 \%)$ & 10 \\
& Baixo & $1(8,3 \%)$ & $2(16,7 \%)$ & $9(75 \%)$ & \multirow{2}{*}{0,256} \\
& Médio & $2(14,3 \%)$ & $0(0 \%)$ & $12(85,7 \%)$ & $0,256)$ \\
\hline \multirow{3}{*}{ Compreensão de textos } & Alto & $1(7,1 \%)$ & $0(0 \%)$ & $13(92,9 \%)$ & \\
& Baixo & $1(8,3 \%)$ & $1(8,3 \%)$ & $10(83,3 \%)$ & \\
& Médio & $1(7,1 \%)$ & $1(7,1 \%)$ & $12(85,7 \%)$ & 0,880 \\
& Alto & $1(7,1 \%)$ & $0(0 \%)$ & $13(92,9 \%)$ & \\
\hline
\end{tabular}

Fonte: As autoras (2020).

Considerando que pessoas com um nível socioeconômico mais alto podem ter acesso a mais profissionais para acompanhamento do caso, era esperado que as crianças provenientes destas famílias do presente estudo alcançassem melhores resultados no que se refere às habilidades de leitura. Os resultados, no entanto, evidenciam que existem mais variáveis envolvidas, visto que apenas o nível socioeconômico da família não parece afetar o resultado obtido na PROLEC.

De fato, Machado e Gonzaga (2007) afirmam que a renda, quando externa, não capta aspectos permanentes do nível socioeconômico da família e há fatores omitidos que simultaneamente afetam sua determinação e o aprendizado das crianças. Os autores constaram, em sua pesquisa sobre o impacto dos fatores familiares sobre a defasagem idade-série de crianças no Brasil, que o público infantil mais vulnerável à ocorrência de idade-série é formado por meninos, moradores de regiões onde há mais dificuldade de oferta educacional e que são pertencentes às famílias maiores, com pais que têm menor nível de instrução, bem como renda familiar menor (MACHADO; GONZAGA, 2007). Em consonância, Feitosa et al. (2005) relatam que, por mais que se encontrem diversos fatores associados às dificuldades na alfabetização inicial, crianças com baixo nível socioeconômico possuem condições ambientais ainda mais restritas para o acesso acadêmico. Tais condições estão relacionadas com a falta de um espaço e de uma rotina mais orientada para o estudo. Os autores complementam dizendo sobre a possibilidade de 
http://dx.doi.org/10.5902/1984686X41426

pais que não podem ou não sabem ajudá-las nas tarefas de casa ou cujas preocupações com a sobrevivência impulsiona a descuidar dessa faceta dos filhos (FEITOSA et al, 2005).

\section{Conclusão}

A avaliação da habilidade de leitura de alunos com TEA com idades entre 8 e 12 anos, matriculados na rede pública de ensino de Curitiba/PR, permitiu observar que a maioria dos alunos possui dificuldade na habilidade da leitura. Nota-se, no entanto, que alguns destes alunos atingem resultados semelhantes aos da amostra normativa do instrumento utilizado para realizar a avaliação da habilidade de leitura, a PROLEC. Esse número é mais expressivo dentre as crianças mais velhas, o que pode sugerir que as crianças que persistem no sistema educacional eventualmente conseguem atingir os resultados esperados no que se refere à habilidade estudada.

Nos testes mais simples, como as provas "Nome ou som das letras" e "Igual-diferente", foi possível observar um melhor desempenho dos participantes. Isso sugere que os alunos com TEA desenvolvem mais facilmente a memorização das letras e palavras do que a compreensão do significado delas, bem como a compressão dos significados das orações que são compostas com essas palavras. Nesse sentido, Cunha (2011) cita que as pessoas com TEA aprendem de forma singular, pois há uma relação diferente entre o cérebro e os sentidos, e as informações nem sempre geram conhecimento. $O$ autor afirma ainda que a concentração para as atividades pedagógicas é muito pequena, contudo, é possível observar resultados mais cedo ou mais tarde (CUNHA, 2011).

Não houve diferença das habilidades de leitura em função do nível socioeconômico da família, o que indica que mais variáveis estão envolvidas no que se refere ao desenvolvimento de habilidades de leitura em crianças com TEA.

As dificuldades no desenvolvimento de habilidades de leitura apresentadas pelas crianças com TEA indicam a necessidade de organização pedagógica para favorecer as habilidades e competências individuais. Nesse sentido, para que não haja desistência diante das dificuldades iniciais, sugere-se que os professores não olhem apenas para os resultados quantitativos dos alunos, mas $\operatorname{sim}$ considerem as capacidades e potencialidades, pois os resultados podem não vir de forma imediata e nem como os educadores esperam. Além disso, entende-se que é de extrema importância reconhecer as diferentes etapas do processo de ensino-aprendizagem, os diferentes processos interdependentes do desenvolvimento das habilidades de leitura, as dificuldades individuais 
de cada um dos seus alunos para que se possa buscar as melhores estratégias disponíveis que favoreçam o processo educacional.

\section{Referências}

AL OTAIBA, Stephanie; FUCHS, Douglas. Characteristics of children who are unresponsive to early literacy intervention: A review of the literature. Remedial and Special Education, v. 23, n. 5, p. 300-316, 2002.

ANDRADE, Elizabete Maria de Almeida. et al. Eficácia de um programa de intervenção fônica para crianças com dificuldades de leitura e escrita. Rev. Psicopedagogia, São Paulo, v. 31, no 95, p. 119-129, 2014.

BRASIL, Constituição da República Federativa do. Texto consolidado até a Emenda Constitucional $\mathbf{n}^{\circ} \mathbf{4 4}$ de $\mathbf{3 0}$ de Junho de 2004. Brasília, p. 54, 2004.

BRASIL. Ministério da Educação. Secretaria da Educação Especial. Política Nacional de Educação Especial na Perspectiva da Educação Inclusiva. Brasília, 2008. Disponível em www.mec.gov.br. Acesso em 13 de novembro de 2017.

BRASIL, Lei ${ }^{\circ} 12.764$ de Dezembro de 2012. Disponível em: http://www.planalto.gov.br/ccivil_03/_ato2011-2014/2012/lei//12764.htm. Acesso em: 28 de Abril de 2016.

BRASIL, Lei $\mathrm{n}^{\circ} \mathbf{1 3 . 1 4 6}$ de Julho de 2015. Disponível em: http://www.planalto.gov.br/ccivil_03/_Ato2015-2018/2015/Lei/L13146.htm. Acesso em: 04 de Maio de 2016.

CUETOS, Fernando; RODRIGUES, Blanca; RUANO, Elvira; adaptação para o português Simone Aparecida Capellini, Adriana Marques de Oliveira e Fernando Cuetos. PROLEC Provas de Avaliação dos Processos de Leitura: manual. São Paulo: Casa do Psicólogo, 2015.

CUNHA, Antônio Egênio. Práticas pedagógicas para a inclusão e diversidade. 2 ed. Rio de Janeiro, Wak Editora, 2011.

DUBET, François. O que é uma escola justa? Cadernos de Pesquisa, v. $34, n^{\circ} 123$, p. 539555, Set./Dez., 2004.

EL ZEIN, Farah. et al. Reading comprehension interventions for students with autism spectrum disorders: A synthesis of research. J Autism Dev Disorder, v. 44, n 6, p. 13031322, 2014.

FEITOSA, Fabio Biasotto. et al. Suporte social, nível socioeconômico e o ajustamento social e escolar de adolescentes portugueses. Temas em Psicologia, v. 13, n 2, p. 129-138, 2005. 
http://dx.doi.org/10.5902/1984686X41426

GOMES, Camila Graciella Santos. Aprendizagem relacional, comportamento simbólico e ensino de leitura a pessoas com transtorno do espectro do autismo. 2011. $176 \mathrm{f}$. Tese (Doutorado) - Universidade Federal de São Carlos, 2011.

GOMES, Camila Graciella Santos. Ensino de leitura para pessoas com autismo. São Paulo: Appris Editora, 2015.

GOMES, Camila Graciella Santos; CARVALHO, Bruna Stella da Silva; SOUZA, Deisy das Graças de. Aspectos relevantes do ensino de leitura para pessoas com transtorno do espectro do autismo. Revista Comportamento em foco, v. 3, p. 59-68, 2014.

GOMES, Camila Graciella Santos; MENDES, Enicéia Gonçalves; Escolarização inclusiva de alunos com autismo na Rede Municipal de Ensino de Belo Horizonte. Revista Brasileira Educação Especial, Marília, v. 16, nº 3, p. 375-396, Set./Dez., 2010.

GOMES, Camila Graciella Santos; SOUZA, Deisy das Graças de. Ensino de sílabas simples, leitura combinatória e leitura com compreensão para aprendizes com autismo. Revista Brasileira de Educação Especial, Marília, v. 22, nº 2, 233-252, Abr./Jun., 2016.

HANNA, Elenice Seixas. et al. Leitura recombinativa de pseudopalavras impressas em pseudoalfabeto: similaridade entre palavras e extensão da unidade ensinada. Revista de Psicologia, São Paulo, v. 21, nº 2, Jun., p. 275-311, 2010.

KERR, Nancy. et al. The measurement of motor, visual and auditory discriminations skills. Rehabilitation Psychology, v. 24, n³, 95-112, 1977.

KRUG, David; ARICK, Joel; ALMOND, Patricia. Behavior checklist for identifying severely handicapped individuals with high levels of autistic behavior. Journal of the American Academy of Child \& Adolescent Psychiatry, v.21, n 3, 221-9, 1980.

MACHADO, Danielle Carusi; GONZAGA, Gustavo. O Impacto dos Fatores Familiares Sobre a Defasagem Idade-Série de Crianças no Brasil. Revista Brasileira de Economia, Rio de Janeiro, v. 61, n 4, p. 449-476, Out./Dez., 2007.

MARTELETO, Márcia Regina Fumagalli; PEDROMÔNICO, Márcia Regina Marcondes. Validity of Autism Behavior Checklist (ABC): preliminary study. Revista Brasileira de Psiquiatria, São Paulo, v. 27, nº 4, 2005.

NATION, Kate. et al. Patterns of reading ability in children with autism spectrum disorder. Journal of Autism and Development Disorders, v. 36, n 7, p. 911-919, 2006.

NATIONAL READING PANEL. National Reading Panel Report: Teaching children to read an evidence based assessment of the scientific research literature on Reading and its implications for Reading instruction. Washington: National Institute of Child Health and Development, 2000. Disponível em:

https://www.nichd.nih.gov/puplications/pubs/nrp/Documents/report.pdf. Acesso em: 11 nov. 2017. 
NUNES, Débora Regina de Paula; AZEVEDO, Mariana Queiroz Orrico; SCHMIDT, Carlo. Inclusão educacional de pessoas com Autismo no Brasil: uma revisão da literatura. Revista Educação Especial, Santa Maria, v. 26, n 47, p. 557-572, Set./Dez., 2013.

NUNES, Débora Regina de Paula; WALTER, Elizabeth Cynthia. Processos de Leitura em Educandos com Autismo: um estudo de revisão. Revista Brasileira de Educação Especial, Marília, v. 22, n4, p. 619-632, Out./Dez., 2016.

OLIVEIRA, Adriana Marques de. et al. Desempenho de escolares com dislexia e transtorno do déficit de atenção e hiperatividade nos processos de leitura. Revista Brasileira:

Crescimento e Desenvolvimento Humano, v. 21, nº 2, p. 344-355, 2011.

OLIVEIRA, Adriana Marques de. et al. Processos de leitura em escolares com Transtorno de Déficit de Atenção/Hiperatividade. Psicologia Argumento, Curitiba, v. 31, n 72, p. 35-44, Jan./Mar., 2013.

PRODANOV, Cleber Cristiano. Metodologia do trabalho científico [recurso eletrônico]: métodos e técnicas da pesquisa e do trabalho acadêmico / Cleber Cristiano Prodanov, Ernani Cesar de Freitas. - 2. ed. - Novo Hamburgo: Feevale, 2013.

SALLES, Jerusa Fumagalli de. et al. Normas de desempenho em tarefa de leitura de palavras/pseudopalavras isoladas (LPI) para crianças de $1^{\circ}$ ano a $7^{\circ}$ ano. Estud. pesqui. psicol. [online]. 2013, vol. 13, n. 2, pp 397-419.

SAMPAIO, Caroline Maria Tavares de; OLIVEIRA, Gislene Farias de. O Desafio da Leitura e da Escrita em Crianças com Perturbações do Espectro do Autismo. Revista de Psicologia, v. 11, n 36, 343-362, 2017.

SANTOS, Myllenna de Oliveira; ZACARIAS, Jaqueline da Cruz; BARBOSA, Amanda; MAGALHÃES, Amanda. Aprendizagem e Transtorno do Espectro Autista - TEA: experiências vivenciadas através do projeto ABC do TRATE. In: I Encontro Nordestino de Inclusão na Educação Superior, v. 1, nº 1, 2015. Anais. Universidade Federal de Alagoas.

SCHOPLER, Eric. et al. Individualized assessment and treatment for autistic and developmentally disabled children: Psychoeducational profile-revised (PEP-R). Autism: PRO-ED, 1990.

SCHOPLER, Eric; REICHLER, Robert Jay; RENNER, Barbara Rochen. CARS - The Childhood Autism Rating Scale. Los Angeles: Western Psychological Services, 1988.

SILVA, Maria da Conceição Lopes da Cruz e. Aprendizagem da Leitura e Escrita em Crianças com Perturbações do Espectro do Autismo: Propostas Pedagógicas. 2011. 110 f. Dissertação (Mestrado) - Faculdade de Artes e Letras, Universidade da Beira Interior, Covilhã 2011.

SILVA, Viviane Freitas da. A presença de alunos autistas em salas regulares, a aprendizagem de ciências e a alfabetização científica: percepções de professores a partir de uma pesquisa fenomenológica. 2016. 187 f. Dissertação (Mestrado) Universidade Estadual Paulista, Faculdade de Ciências - Bauru, 2016. 
http://dx.doi.org/10.5902/1984686X41426

SKINNER, Burrhus Frederic. Tecnologia do ensino. (Rodolpho Azzi, Trad.). São Paulo: Herder, Ed. da Universidade de São Paulo 1972.

TALARICO, Mariana Valente Teixeira da Silva; LAPLANE, Adriana Lia Friszman de. Trajetória escolar de alunos com Transtorno do Espectro Autista. Comunicações Piracicaba, v. 23, n. 3, p. 43-56, 2016.

TAMANAHA, Ana Carolina; MARTELETO, Marcia Regina Fumagalli; PERISSINOTO, Jacy. A intervenção do status de linguagem expressiva na pontuação do Autism Behavior Checklist em autistas verbais e não verbais. Audiol Commun Res., v. 19, $n^{\circ}$ 2, 167-170, 2014.

UNESCO. Declaração de Salamanca. Sobre Princípios, Políticas e Práticas na Área das Necessidades Educativas Especiais, 1994. Disponível em:

http://portal.mec.gov.br/seesp/arquivos/pdf/salamanca.pdf. Acesso em: 28 ago. 2020.

\section{Notas}

${ }^{1} \mathrm{Na}$ tradução para o português do DSM-5 (APA, 2014), Austism Spectrum Disorder é apresentado como Transtorno do Espectro Autista. No presente estudo, adotamos a denominação Transtorno do Espectro do Autismo por considerarmos que esse termo deixa claro que são diferentes apresentações do mesmo transtorno.

2 Cuetos, Rodrigues e Ruano (2015).

\section{Correspondência}

Karize Younes Barberini Perim - Universidade Presbiteriana Mackenzie, R. da Consolação, 930 - Consolação, São Paulo, São Paulo - Brasil.

CEP: 01302-907

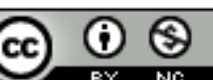

4.0 International (CC BY-NC 4.0) 